\title{
Flux pump for HTS rotating machinery applications
}

\author{
Ravichandra Kulkarni ${ }^{1}$, Krishnamachar Prasad, Tek Tjing Lie
}

School of Engineering, Auckland University of Technology, Private Bag 92006, Auckland 1142 ('email:rkulkarn@aut.ac.nz).

\begin{abstract}
High Temperature Superconducting (HTS) machines offer several benefits over the conventional machines. With the advent of YBCO materials, research and development in HTS applications has gained significant impetus. The application of flux pump has its own advantages over the conventional mechanism for magnetic field generation in superconductors. This paper reviews few relevant works and presents preliminary results from experiments carried out to provide justification on the application of flux pump for generating magnetic flux in a rotating machine.
\end{abstract}

Keywords: High Temperature Superconductors (HTS), Low Temperature Superconductors (LTS), YBCO (Yttrium Barium Cupric Oxides), Flux pump, Superconducting generator

\section{INTRODUCTION}

(a) HTS materials

High temperature superconductivity has been the limelight of research groups in the domain of superconductivity. HTS materials have evolved to a great extent since their discovery and few of the applications incorporating their outstanding features have reached commercial stage [1]. Taking cryogenics into account, these materials can be suitably used, with a tradeoff between size and efficiency. HTS materials exhibit superconductivity at relatively high temperatures ( $>35 \mathrm{~K}$ ) compared to the LTS materials $(<35 \mathrm{~K})[1,2]$.

Design of magnets using LTS is an old technology and dominates the Magnetic Resonance Imaging (MRI) applications to date. HTS magnets have also been designed and have proven to trap high strength magnetic fields [3].The high current densities $\left(\sim 106-108 \mathrm{~A} / \mathrm{m}^{2}\right)$ and high magnetic field capabilities (4 - $5 \mathrm{~T}$ ) of the $2 \mathrm{G}$ HTS (YBCO) wires make them outstandingly 'super' [3-5].

\section{(b) HTS rotating machinery and Thermal loading}

HTS based generators are predicted to capture $50 \%$ of the present market of rotating electrical machines by the year 2020 [6]. As the year is nearing, it will be socially beneficial if this prediction turns out to be a reality.

Low weight, small size and high efficiency are the characteristics of superconducting generators with LTS materials. Various types have been studied since 1960s [7]. Seemingly, HTS based rotating machinery are new and possess better commerciality than LTS machines $[2,8,9]$.
Reliance Electric/Rockwell Automation is reported to be the first corporation to have started development of HTS motors in the United States. A 200-hp motor was built and tested in 1996, followed by a larger 1000-hp motor tested in year 2000. Both motors were four-pole $60 \mathrm{~Hz}$ using conventional copper armatures with HTS field coils [2]. In 2002, Siemens developed and presented results of $380 \mathrm{~kW}$ synchronous machine with HTS rotor windings.[10].

Kalsi et al. [2] discuss different topologies of superconducting generator for direct driven wind turbines. One of the claims in this work is that when referred to overall size, efficiency and drivetrain costs, the HTS generator concept is superior to permanent magnet direct drive generator.

Application of HTS materials in the design of rotating machinery puts in the advantages of the HTS materials into the rotating machinery but also poses its own design challenges.

One of the common concern of most of the work on HTS rotating machines is the thermal interaction between the cryogenic and non-cryogenic parts of the system. This interaction loads the cryogenic system with thermal load and results in good amount of loss and reduces overall efficiency of the system[11].

\section{(c) Flux pump}

The feasibility of superconductor applications is dependent on the heat leak produced by current feed-through and the dissipation in the normal parts of the current leads. For commercial operation of superconducting systems, reduction of these losses is inevitable. Flux pump is one potential solution for this problem $[12,13]$.

Flux pump offers a unique method of magnetizing a superconductor without a physical link between the cryogenic and non-cryogenic environment. If suit-fitted properly for an application, it can resolve the problem of thermal link between the cryogenic and non-cryogenic environment.

Application of a mechanically rotating flux pump to energize an HTS magnet with a current ramp up rate of $0.44 \mathrm{~A} / \mathrm{s}$ with a maximum current of $\quad 49 \mathrm{~A}$ in a $2.7 \mathrm{mH}$ HTS coil has been successfully demonstrated. It is also observed that the rate of rise of current is dependent on the number of tapes and also on the frequency of rotation (Fig 1) of the flux pump [14].

This flux pump operates and flux is pinned into the superconductors without a switching circuit as in a conventional flux pump application. 


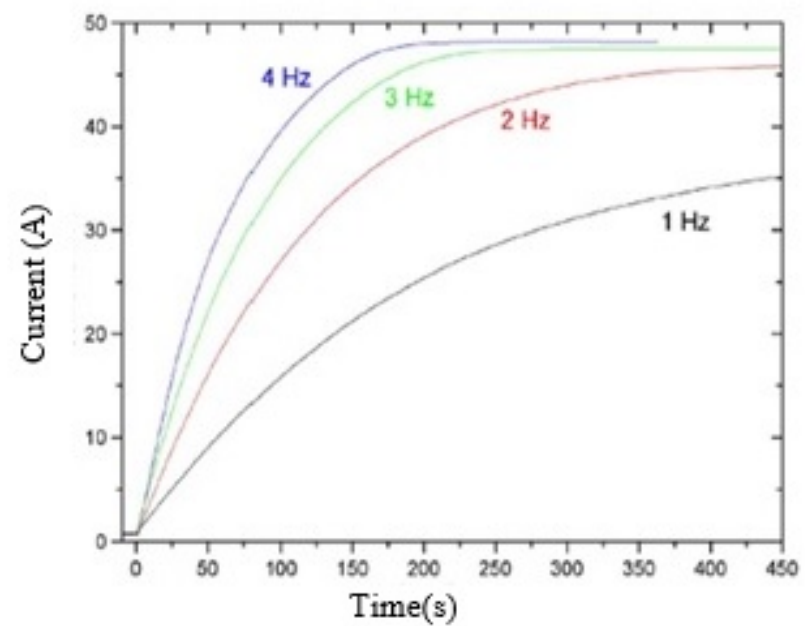

Fig 1. Current ramp up in a HTS tape with different frequencies of flux interaction as observed by Hoffmann et al.., [14].

A thermally actuated flux pump has been proposed by Coombs et $a l$., and the technique used enables the creation of a high magnetic field limited by the characteristic features of the super conductor and not the magnetizing system [15]. The method applied here acts on the surface of the superconductor and offers shaping and patterning of the magnetic field with use of multiple pumps on the surface of superconductor.

The authors, however, have not commented on the suitability of this method to a HTS rotating machine. Though it is worth investigating, we believe a simpler method would conveniently solve the issue and this will be discussed later.

\section{FLUX PUMP AND HTS ROTATING MACHINERY}

One of the complex design challenges is to maintain isolation between the cryogenic and non-cryogenic environment in a superconducting application. This becomes far more complex when the superconducting application involves a dynamic component. A HTS synchronous generator is one such application. The excitation system of the available HTS generators use current leads and these current leads create a thermal link between the cryogenic and non-cryogenic environment.

Work on improving the current leads from cryogenic environment to non-cryogenic environment has been carried out [11]. Method of thermal anchoring of the conductioncooled current leads reports a decrease in cooling load on the cryogenic system. Despite having reduced the load on the cryocooler, a thermal link still exists and loading does occur in this system.
A conceptual design on use of flux pump for rotor excitation is proposed in [17] but no further work on the practicality of the design and applicability of the design has been reported.

\section{EXPERIMENTS AND OBSERVATIONS}

Formulation of dynamic resistance model for a high temperature superconducting flux pump has been presented [16]. The current and voltage profiles with a gap of $1 \mathrm{~mm}$, reported in [16]. The results show a linear agreement between the frequency of the flux pump and the rate of current increase. From the voltage profile, it is evident that a net voltage (quasi DC emf) drop occurs across the superconducting coil.

The time dependent current $I(t) \quad(<I c$, critical current) has been derived in previous works [16] and is given by

$$
I(t)=\frac{\Delta \phi}{\beta}\left(1-\exp \left(\frac{-\beta f t}{L}\right)\right)
$$

where, $\Delta \phi=$ the net flux change

$$
\begin{aligned}
& \beta=\text { constant defined by the design parameters } \\
& \text { of the flux pump }
\end{aligned}
$$

Experiments were carried out by us on an HTS flux pump, with mechanical rotor that contained $12 \mathrm{NdFeB}$ magnets and a type II HTS stator. Figure 2 illustrates the interaction between the magnetic field lines and the HTS stator. When the magnet moves with respect to the HTS stator in the XZ-plane, the flux lines are cut by the HTS stator and a current is induced in the HTS stator.

Type I superconductor repels all the magnetic lines (Meissner effect) when placed in a magnetic field and the magnetic field strength is below critical field for the superconductor, whereas type II superconductor operates in a mixed state when the applied magnetic field strength lies in between the $1^{\text {st }}$ critical field and $2^{\text {nd }}$ critical field thus causing an induced emf and current in the HTS stator.

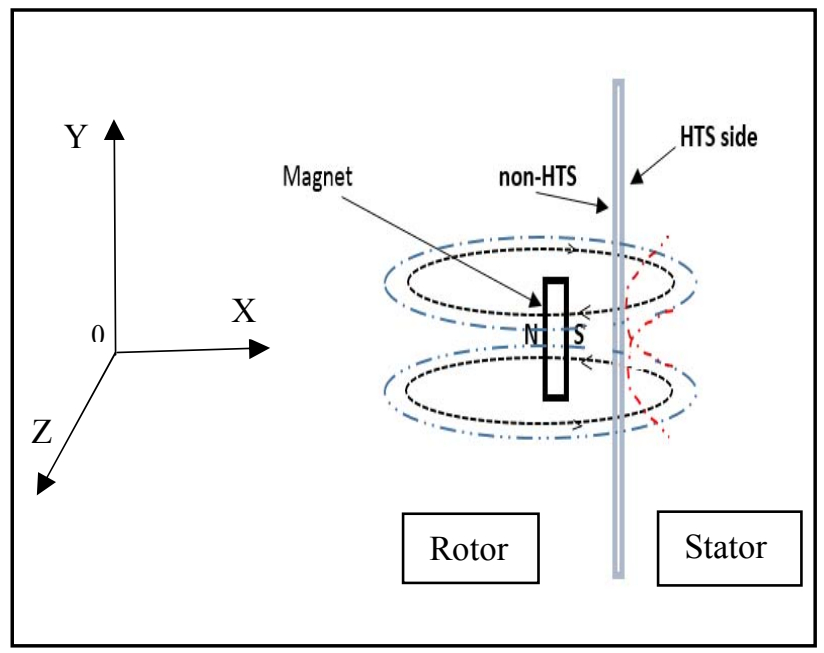

Fig 2. Flux interaction between the rotor magnets and HTS stator. 
The current in the HTS stator was measured using a solenoid coil and Hall sensor arrangement. The hall sensor senses the variation in the magnetic field and provides a proportional voltage as output. This voltage is in the range of millivolts. Lettech amplifier was used to amplify the hall voltages.

A high rate data acquisition system was designed using LABVIEW and NI-DAQ cards. The frequency of data acquisition was selected based on the frequency of flux interaction between the rotor and the stator of the flux pump.

The amplified hall voltage signals were acquired by LABVIEW program via NI-DAQ cards and stored in TDMS file format to facilitate large data management.

In the experiment, the speed $N$ of the flux pump rotor was set as per the following equation;

$$
N=(60 * f) / P \text {------------------2 }
$$

where,

$$
\begin{aligned}
& f=\text { frequency }(\mathrm{Hz}) \\
& P=\text { Number of permanent magnets }
\end{aligned}
$$

In order to avoid resonance with the line voltage, the frequency was set at $598 \mathrm{~Hz}$ and the corresponding speed was set at 2990 rpm for a $12 \mathrm{NdFeB}$ magnets rotor. The speed of the rotor was controlled and varied using OMRON Servo driver and TRIO Motion Coordinator.

The results observed are in agreement with the previous work [16]. The voltage waveform across the HTS stator of flux pump is shown in Fig 3. The voltage across the HTS stator is asymmetric over the zero reference line.The averaging of the voltage profile is shown in Fig. 4 and gives a non-zero DC value, hence justifying a quasi-DC emf across the HTS stator.

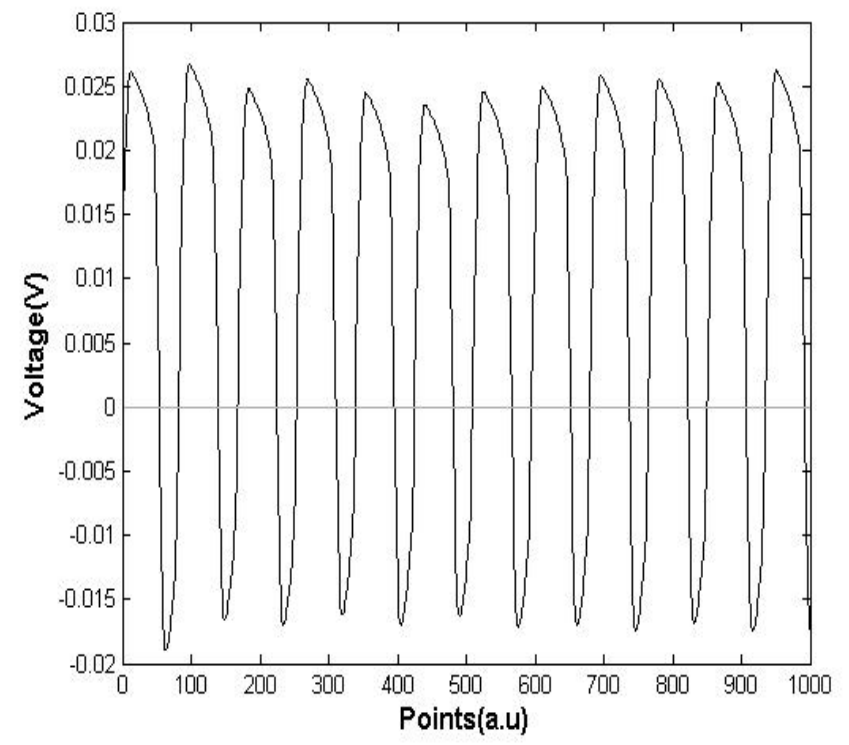

Fig 3. Voltage waveform $(598 \mathrm{~Hz})$ across the HTS stator during flux pump operation.

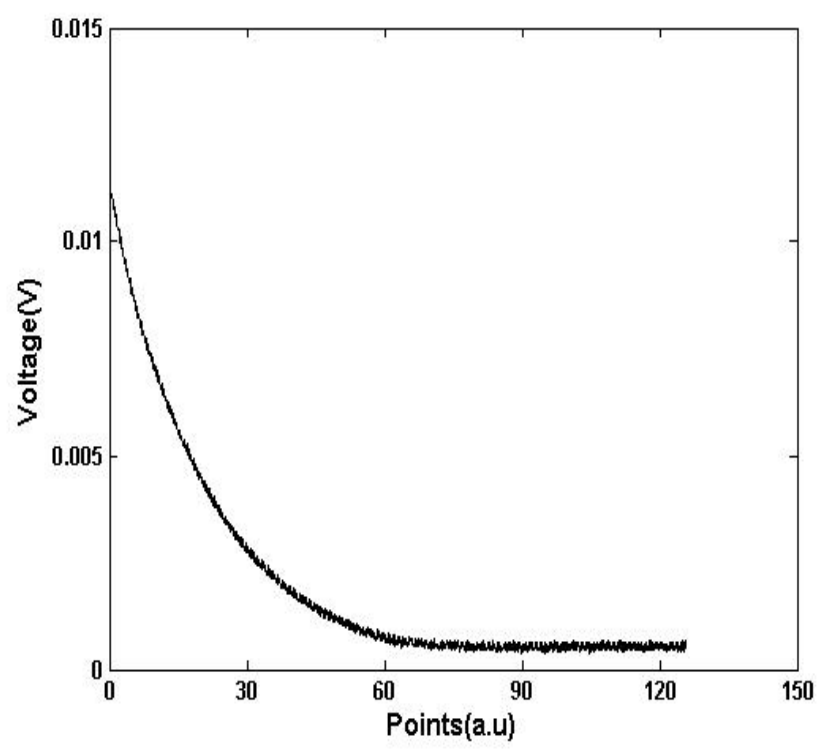

Fig 4. Averaged voltage $(598 \mathrm{~Hz})$. The averaging period was $\sim 5000$ data points.

The average voltage across the HTS stator once the current reaches its saturation is non-zero and is $\sim 0.6 \mathrm{mV}$ for $1 \mathrm{~mm}$ separation at $598 \mathrm{~Hz}$.

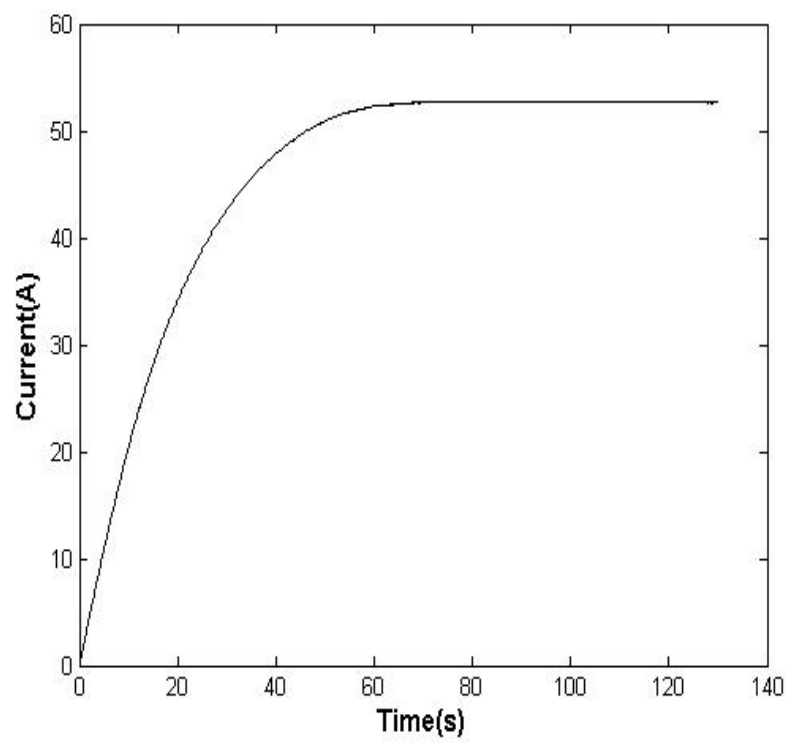

Fig 5. The current ramp up for $1 \mathrm{~mm}$ separation and $598 \mathrm{~Hz}$.

The current ramp up for $1 \mathrm{~mm}$ separation and $598 \mathrm{~Hz}$ frequency is shown in Fig 5. The ramp up rate is around 0.875 $\mathrm{A} / \mathrm{s}$. The maximum current reached is $52.5 \mathrm{~A}$. The steady state is reached in $65 \mathrm{~s}$. 


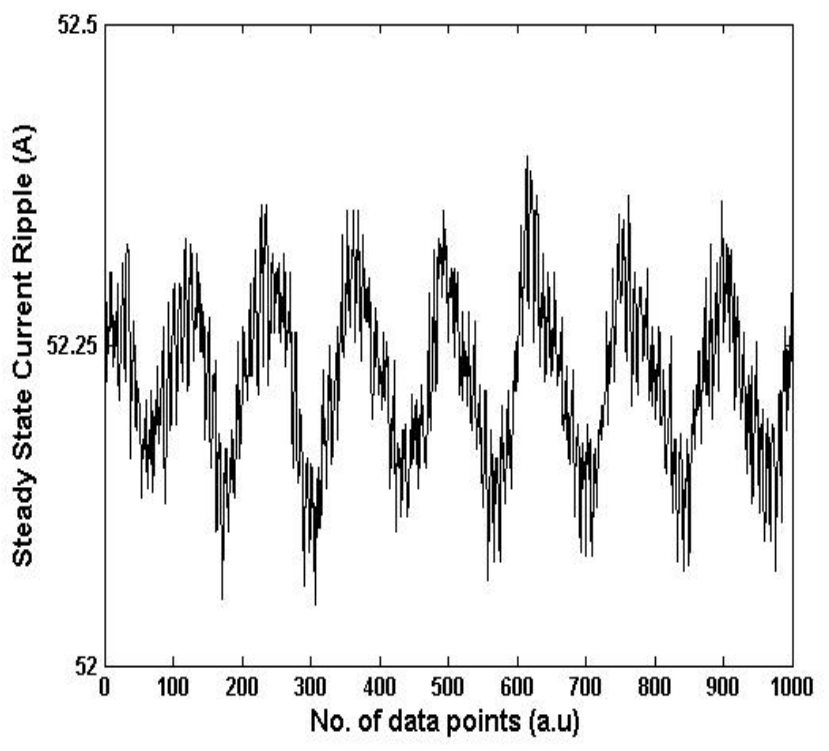

Fig 6. Steady state current ripple $(598 \mathrm{~Hz})$.

From the current ripple, as shown in Fig 6, it can be inferred that the field fluctuation would be similar to the current ripple as the field due to a current carrying conductor is proportional to the current flowing through it. Further experimentation and Finite Element Modelling (FEM) studies are needed to critically analyze the field pattern and fluctuations in the field taking into account the shielding currents, demagnetizing and cross magnetizing effects due to stator conductor field.

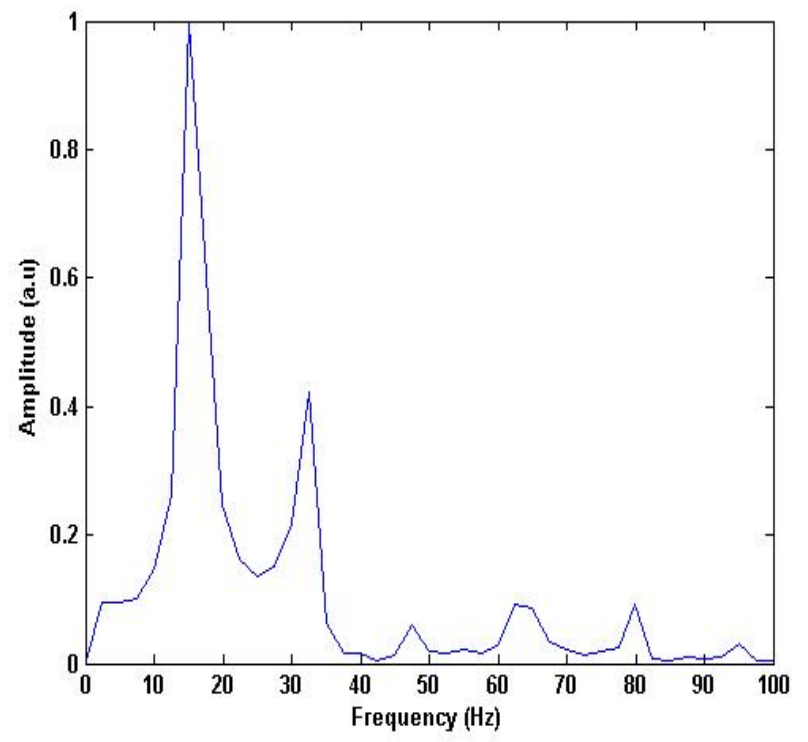

Fig 7. Normalized FFT of the Voltage $(16 \mathrm{~Hz})$ across the HTS stator.
The FFT of the voltage across HTS stator is shown in the Fig 7.

The normalized FFT of the voltage signal across the HTS stator showed the presence of $2^{\text {nd }}$ and $4^{\text {th }}$ harmonics. The presence of even harmonics are because of the asymmetry in the voltage and this has never been reported. However, an interesting factor is the magnitude of the harmonics.

The magnitude of $2^{\text {nd }}$ and $4^{\text {th }}$ harmonics are observed to be $30 \%$ and $4 \%$ of the fundamental, respectively. The $3^{\text {rd }}$ harmonic is found to be smaller than the $4^{\text {th }}$ harmonic.

Further investigation and analysis with a larger data set is currently in progress to understand the nature and magnitude of various harmonics in the voltage profile.

\section{CONCLUSION}

From the results of our flux pump experiments in magnetizing a superconductor, it is clear that the flux pump can be utilized in generating magnetic field in an HTS rotating machinery.

Some of the concerns that need to be addressed such as:

a) An efficient magnetic circuit to bridge the magnetic flux between flux pump magnets and the superconductor.

b) Harmonic analysis of the system in conjunction with the synchronous generator operating conditions.

c) Demagnetizing and cross-magnetizing effects of armature conductors on the flux link between flux pump magnets and superconductor.

An empirical formula for the rate of rise of current and its dependency on the shape and size of the magnets used in the flux pump and choice of material of the magnetic circuit are some of the aspects for improvising the design of the flux pump in order to suit-fit it to a rotating machinery application.

Based on the earlier reported works and preliminary results of our work, it can be concluded that application of flux pump is suitable for exciting rotor (HTS conductors) in a synchronous generator. Use of flux pump would reduce the thermal load on the cryogenic system of the HTS generator and improve the commercial applicability, an improvement in the efficiency of the system can also be expected.

\section{ACKNOWLEDGMENTS}

The authors would like to thank Rod Badcock, Chris Bumby and Zhenan Jiang of Robinson Research Institute, Victoria University of Wellington, for their cooperation and help in carrying out this study. 


\section{REFERENCES}

[1] S. S. Kalsi, Dave Gritter, Om Nayak and Chuck Gallagher, "Benefits of HTS technology to ship systems " presented at the IEEE Symposium on Electric Ship Technologies, Philadelphia PA, 2005.

[2] S. S. Kalsi, K. Weeber, H. Takesue, C. Lewis, H. W. Neumueller, and R. D. Blaugher, "Development status of rotating machines employing superconducting field windings," Proceedings of the IEEE, vol. 92, pp. 1688-1704, 2004.

[3] K. T. A. M. Murakami, "High-temperature superconductor bulk magnets that can trap magnetic fields of over 17 Tesla at 29 K," Nature, p. 4, 2003.

[4] S. Kalsi, Application of High Temperature Superconductors to Electric Power Equipment: Wiley, 2011.

[5] P. N. Barnes, M. D. Sumption, and G. L. Rhoads, "Review of high power density superconducting generators: Present state and prospects for incorporating YBCO windings," Cryogenics, vol. 45, pp. 670-686, 10// 2005.

[6] T. P. S. A. B. M. Joseph Mulholland, "Analysis of Future Prices and Markets for High Temperature Superconductors," U.S. Department of EnergyJune 2003.

[7] Q. Ronghai, L. Yingzhen, and W. Jin, "Review of Superconducting Generator Topologies for DirectDrive Wind Turbines," Applied Superconductivity, IEEE Transactions on, vol. 23, pp. 52011085201108, 2013.

[8] B. B. Jensen and A. B. Abrahamsen, "Modeling, construction, and testing of a simple HTS machine demonstrator," in Energy Conversion Congress and Exposition (ECCE), 2011 IEEE, 2011, pp. 16361640.

[9] Z. Min, W. Wei, Y. R. Chen, and T. Coombs, "Design Methodology of HTS Bulk Machine for

\section{About Authors:}

Ravichandra Kulkarni received B.E degree in Electrical and Electronics from Visvesvaraya Technological University, Belgaum, India, in 2008, M.Tech degree in Industrial Electronics in 2010. He is currently pursuing research in the field of high temperature superconductor applications for rotating electrical machines.

Krishnamachar Prasad (M'08, SM'11) received the B.E. degree from Bangalore University, Bangalore, India, in 1980 , the M.Tech. degree in electrical engineering from the Indian Institute of Technology, Madras, India, in 1982, and the Ph.D. degree from the University of Western Australia, Perth, Australia, in 1990. He is currently a Professor with the Department of Electrical and Electronic Engineering, Auckland University of Technology, Auckland, New Zealand. His research interests include material science,
Direct-Driven Wind Generation," Applied Superconductivity, IEEE Transactions on, vol. 22, pp. 5201804-5201804, 2012.

[10] W. Nick, G. Nerowski, H. W. Neumüller, M. Frank, P. van Hasselt, J. Frauenhofer, et al., "380 kW synchronous machine with HTS rotor windingsdevelopment at Siemens and first test results," Physica C: Superconductivity, vol. 372-376, Part 3, pp. 1506-1512, 8// 2002.

[11] S. Jeong and Y. Kim, "Thermal anchoring of conduction-cooled current leads for superconductivity applications near liquid nitrogen temperature," Cryogenics, vol. 50, pp. 287-291, 4// 2010.

[12] L. J. M. van de Klundert and H. H. J. ten Kate, "Fully superconducting rectifiers and fluxpumps Part 1: Realized methods for pumping flux," Cryogenics, vol. 21, pp. 195-206, 4// 1981.

[13] C. Hoffmann, R. Walsh, E. Karrer-Mueller, and D. Pooke, "Design Parameters for an HTS Flux Pump," Physics Procedia, vol. 36, pp. 1324-1329, // 2012.

[14] C. Hoffmann, D. Pooke, and A. D. Caplin, "Flux Pump for HTS Magnets," Applied Superconductivity, IEEE Transactions on, vol. 21, pp. 1628-1631, 2011.

[15] T. Coombs, Z. Hong, and X. Zhu, "A thermally actuated superconducting flux pump," Physica $C$ : Superconductivity, vol. 468, pp. 153-159, 2/1/ 2008.

[16] Z. Jiang, K. Hamilton, N. Amemiya, R. A. Badcock, and C. W. Bumby, "Dynamic resistance of a highTemperature superconducting flux pump," Applied Physics Letters, vol. 105, pp. 112601-112601-4, 2014.

[17] Ishmael, S., Goodzeit, C., Masson, P., Meinke, R., and Sullivan, R. (2008). Flux Pump Excited DoubleHelix Rotor for Use in Synchronous Machines. Applied Superconductivity, IEEE Transactions on, 18(2), 693-696. doi: 10.1109/TASC.2008.922545

microelectronic devices, and failure analysis and reliability engineering.

Tek Tjing Lie (S'89-M'92-SM'97) received the B.S. degree from Oklahoma State University, Stillwater, in 1986 and the M.S. and Ph.D. degrees from Michigan State University, East Lansing, in 1988 and 1992, respectively. $\mathrm{He}$ is currently a Professor with the Department of Electrical and Electronic Engineering, Auckland University of Technology, Auckland, New Zealand. His research interests include power system control, deregulated power systems, and renewable energy systems. 